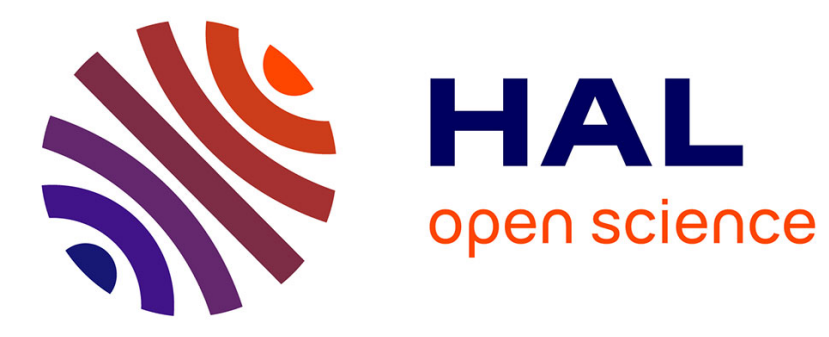

\title{
Qui sème la restriction récolte la récession
}

Céline Antonin, Hervé Péléraux, Mathieu Plane, Xavier Timbeau

\section{To cite this version:}

Céline Antonin, Hervé Péléraux, Mathieu Plane, Xavier Timbeau. Qui sème la restriction récolte la récession: Perspectives économiques 2012-2013 pour l'économie mondiale. OFCE Les notes du blog, 2012, 16, pp.1-22. hal-01053555

\section{HAL Id: hal-01053555 \\ https://hal-sciencespo.archives-ouvertes.fr/hal-01053555}

Submitted on 31 Jul 2014

HAL is a multi-disciplinary open access archive for the deposit and dissemination of scientific research documents, whether they are published or not. The documents may come from teaching and research institutions in France or abroad, or from public or private research centers.
L'archive ouverte pluridisciplinaire HAL, est destinée au dépôt et à la diffusion de documents scientifiques de niveau recherche, publiés ou non, émanant des établissements d'enseignement et de recherche français ou étrangers, des laboratoires publics ou privés. 


\title{
les notes
}

$N^{\circ} 16$ / 29 mars 2012

\section{Qui sème la restriction récolte la récession Perspectives économiques 2012-2013 pour l'économie mondiale}

\author{
Département analyse et prévision 1, \\ sous la direction de Xavier Timbeau
}

- paroxysme paroxysme de la crise des dettes souveraines est passé. La dette publique grecque est restructurée et diminuera, au prix d'un défaut partiel, de 160 à $120 \%$ du PIB. Cette restructuration autorise le déblocage du soutien financier de la troïka à la Grèce et résout pour l'instant le problème de financement du renouvellement de la dette publique grecque. La contagion qui avait frappé la plupart des pays de la zone euro, et qui s'était traduite par une hausse des taux souverains, est interrompue. La détente est sensible par rapport au début de l'année 2012 et le risque d'un éclatement de la zone euro est largement réduit, du moins dans le court terme.

Pour autant, le processus de transformation de la Grande Récession, amorcée en 2008, en très Grande Récession ${ }^{2}$ n'est pas interrompu par le soulagement temporaire apporté à la crise grecque. D'une part, l'économie mondiale, et singulièrement la zone euro, restent dans une zone à risque où, à nouveau, une crise systémique menace et d'autre part, la stratégie choisie par l'Europe, à savoir la réduction rapide de la dette publique (qui suppose la réduction des déficits publics et leur maintien en deçà des déficits qui stabilisent la dette) compromet l'objectif annoncé. Or, puisque la crédibilité de la stratégie est une condition indispensable en zone euro pour rassurer les marchés financiers, et permettre le financement à un taux acceptable des besoins de finance-

1. Ce texte synthétise l'analyse de la conjoncture menée par le Département analyse et prévision de l'OFCE au printemps 2012. II a été rédigé par Céline Antonin, Hervé Péléraux, Mathieu Plane et Xavier Timbeau. II s'appuie sur le travail d'une équipe dirigée par Xavier Timbeau et composée de Céline Antonin, Christophe Blot, Marion Cochard, Bruno Ducoudré, Amel Falah, Éric Heyer, Sabine Le Bayon, Catherine Mathieu, Hervé Péléraux, Mathieu Plane, Christine Rifflart et Danielle Schweisguth. II intègre les informations disponibles au 27 mars 2012.

2. Voir « La très Grande Récession : perspectives économiques mises à jour pour les grands pays développés en 2012 ", sous la direction de Xavier Timbeau, Département analyse et prévision, Les Notes de l'OFCE, n 9, 16 décembre 2011. 
ment de la dette publique (entre 10 et $20 \%$ de cette dette étant refinancés chaque année), la difficulté à atteindre l'objectif oblige à une rigueur toujours plus grande. La zone euro apparaît comme courant après une stratégie dont elle ne maîtrise pas les leviers, ce qui ne peut qu'alimenter la spéculation et l'incertitude.

Nos prévisions pour la zone euro sont d'une baisse du PIB de 0,4 point en 2012 et d'une croissance de 0,3 point en 2013 (tableau 1). Le PIB par tête baisserait en 2012 pour la zone euro et serait stable en 2013. Le Royaume Uni échapperait à la récession en 2012, mais la croissance du PIB resterait en 2012 et en 2013 en deçà de 1 \% par an. Aux États-Unis, la croissance du PIB accélèrerait à 2,3\% par an en 2012 après une année 2011 à 1,7\%. Bien qu'il soit supérieur à celui de la zone euro, ce taux de croissance peine à enclencher une hausse du PIB par tête et ne permet pas une décrue significative du chômage.

L'épicentre de la crise se déplace ainsi vers le Vieux continent et compromet la sortie de crise pour tous les pays développés. Confrontés, plus encore que la zone euro, à une situation budgétaire dégradée et donc à l'alourdissement de la dette (tableau 3), les États-Unis et le Royaume-Uni sont également pris dans le pari sur la soutenabilité de leur dette publique. La restriction budgétaire en zone euro pèse sur leur activité et ne fait qu'accroître leurs difficultés.

Si l'activité peine à retrouver son niveau antérieur, le «new normal », c'est-à-dire la nouvelle trajectoire de l'activité résultant de l'impact durable et permanent de la crise, s'imposera comme le scénario réaliste. C'est ce que suggère par exemple l'analyse historique conduite par le $\mathrm{FMI}^{3}$ où il apparaît que les crises bancaires réduisent de façon permanente le niveau de l'activité de presque 10 points. L'extrapolation de cette analyse à la crise actuelle ne saurait cependant être faite trop rapidement. La preuve empirique apportée par l'analyse historique est faible d'autant qu'elle inclut dans l'échantillon de nombreux «petits » pays. II est difficile par exemple de conclure à un impact permanent de la Grande Dépression des années 1930, même si la prospérité n'est revenue que longtemps après le déclenchement de la crise.

Les trimestres qui viennent seront décisifs pour mesurer l'ampleur de l'ajustement à réaliser. Si le « new normal » est la règle, alors il faut, pour retrouver un équilibre des finances publiques, un ajustement de grande ampleur. Les écarts à la production tendancielle sont de l'ordre de 5 à 10 points selon les pays et les déficits structurels «new normal » du même ordre. En revanche, si l'impact est moins important, ou s'il n'y a pas d'impact permanent, alors l'ajustement structurel à réaliser est bien moindre. Le graphique 1 illustre ces possibilités pour la France. Une approche similaire peut être conduite pour tous les pays développés.

En privilégiant la réduction rapide des déficits et de la dette publique, les décideurs de la zone euro révèlent que leur croyance dans le futur est le scénario du pire. Reposer sur la soi-disant discipline des marchés pour rappeler à l'ordre les pays dont les finances publiques sont dégradées ne fait qu'accroître par le jeu du renchérissement des taux d'intérêts souverains le problème de soutenabilité. Or, en induisant par un effet multiplicateur toujours sous-estimé dans l'élaboration des stratégies ou des prévisions (encadré) une moindre activité, cette croyance résignée dans un «new normal » dégradé semble se confirmer. In fine, elle ne fait que s'auto-réaliser.

3. Zdzienicka, A. et Furceri, D. (n.d.), « How Costly are Debt Crises? », papers.ssrn.com 
Qui sème la restriction récolte la récession : Perspectives économiques 2012-2013

Tableau 1. Perspectives de croissance mondiale

Taux de croissance annuels, en \%

\begin{tabular}{|c|c|c|c|c|}
\hline & \multirow{2}{*}{$\begin{array}{c}\text { Poids }{ }^{1} \\
\text { dans le total }\end{array}$} & \multicolumn{3}{|c|}{ PIB en volume } \\
\hline & & 2011 & 2012 & 2013 \\
\hline Allemagne & 4,2 & 3,1 & 0,3 & 0,8 \\
\hline France & 3,1 & 1,7 & 0,2 & 0,7 \\
\hline Italie & 2,6 & 0,5 & $-1,7$ & $-0,9$ \\
\hline Espagne & 2,0 & 0,7 & $-1,1$ & $-0,6$ \\
\hline Pays-Bas & 1,0 & 1,3 & $-1,1$ & 0,5 \\
\hline Belgique & 0,6 & 1,9 & 0,1 & 0,9 \\
\hline Autriche & 0,5 & 3,1 & 0,4 & 0,8 \\
\hline Finlande & 0,5 & 2,7 & 0,7 & 1,1 \\
\hline Portugal & 0,3 & $-1,5$ & $-2,9$ & 0,2 \\
\hline Grèce & 0,3 & $-6,2$ & $-5,3$ & $-0,3$ \\
\hline Irlande & 0,3 & 0,7 & $-0,3$ & 0,9 \\
\hline Zone euro & 15,5 & 1,5 & $-0,4$ & 0,3 \\
\hline Royaume-Uni & 3,2 & 0,9 & 0,7 & 0,9 \\
\hline Suède & 0,5 & 4,0 & 0,8 & 1,6 \\
\hline Danemark & 0,3 & 1,1 & 0,6 & 1,1 \\
\hline Europe à 15 & 19,4 & 1,5 & $-0,2$ & 0,4 \\
\hline 12 nouveaux pays membres & 2,7 & 3,1 & 1,3 & 2,1 \\
\hline Europe à 27 & 22,0 & 1,7 & $-0,1$ & 0,6 \\
\hline Suisse & 0,5 & 1,9 & 0,2 & 1,0 \\
\hline Norvège & 0,4 & 2,5 & 2,3 & 2,8 \\
\hline Europe & 22,9 & 1,8 & 0,0 & 0,6 \\
\hline États-Unis & 20,8 & 1,7 & 2,3 & 2,4 \\
\hline Japon & 6,3 & $-0,6$ & 1,9 & 1,5 \\
\hline Canada & 1,9 & 2,3 & 2,0 & 2,3 \\
\hline Pays industriels & 53,5 & 1,4 & 1,2 & 1,5 \\
\hline Pays candidats à I'UE 2 & 1,5 & 7,6 & 1,7 & 4,3 \\
\hline Russie & 3,3 & 4,3 & 3,5 & 3,8 \\
\hline Autres $\mathrm{CEI}^{3}$ & 1,3 & 5,9 & 3,7 & 4,2 \\
\hline Chine & 11,5 & 9,2 & 8,3 & 8,1 \\
\hline Autres pays d'Asie & 13,2 & 5,6 & 5,8 & 6,3 \\
\hline Amérique latine & 8,7 & 4,3 & 3,3 & 3,5 \\
\hline Afrique subsaharienne & 2,3 & 4,9 & 5,5 & 5,3 \\
\hline Moyen-Orient et Afrique du nord & 4,8 & 3,1 & 3,2 & 3,6 \\
\hline Monde & 100 & 3,5 & 3,1 & 3,4 \\
\hline
\end{tabular}

1. Pondération selon le PIB et les PPA de 2008 estimés par le FMI.

2. Croatie, République de Macédoine et Turquie.

3. Communauté des États indépendants.

Sources : FMI, OCDE, sources nationales, calculs et prévision OFCE mars 2012. 
Ces ajustements ne laisseront pas la croissance indemne, et le retournement conjoncturel de la mi-2011, qui paraît engager la zone euro sur la voie d'une nouvelle récession, rappelle les dangers qu'il y aurait à sous-estimer leur l'impact. On peut escompter des effets multiplicateurs d'autant plus intenses que les restrictions budgétaires sont menées dans une situation conjoncturelle dégradée, avec des écarts de production qui restent importants et un niveau de chômage élevé, sans que les pays émergents ne puissent réellement amortir ces chocs négatifs en 2012 et en 2013. La résistance à la baisse des ratios budgétaires pourrait alors conduire à une surenchère dans la restriction pour rassurer les marchés. Si les gouvernements voulaient tenir coûte que coûte des objectifs inatteignables pour la majorité d'entre eux, le choc sur l'activité serait puissant, accompagné d'une récession violente et faisant réapparaître le risque d'un nouveau défaut dans la zone euro. Et même à supposer que les efforts consentis en Europe s'avèrent payants, le risque se déporterait de la zone euro vers les pays anglo-saxons qui pourraient passer, compte tenu de situations budgétaires toujours tendues auxquelles il n'aura pas vraiment été porté remède, dans le viseur des marchés.

Graphique 1. Après la crise ou le « new normal »?

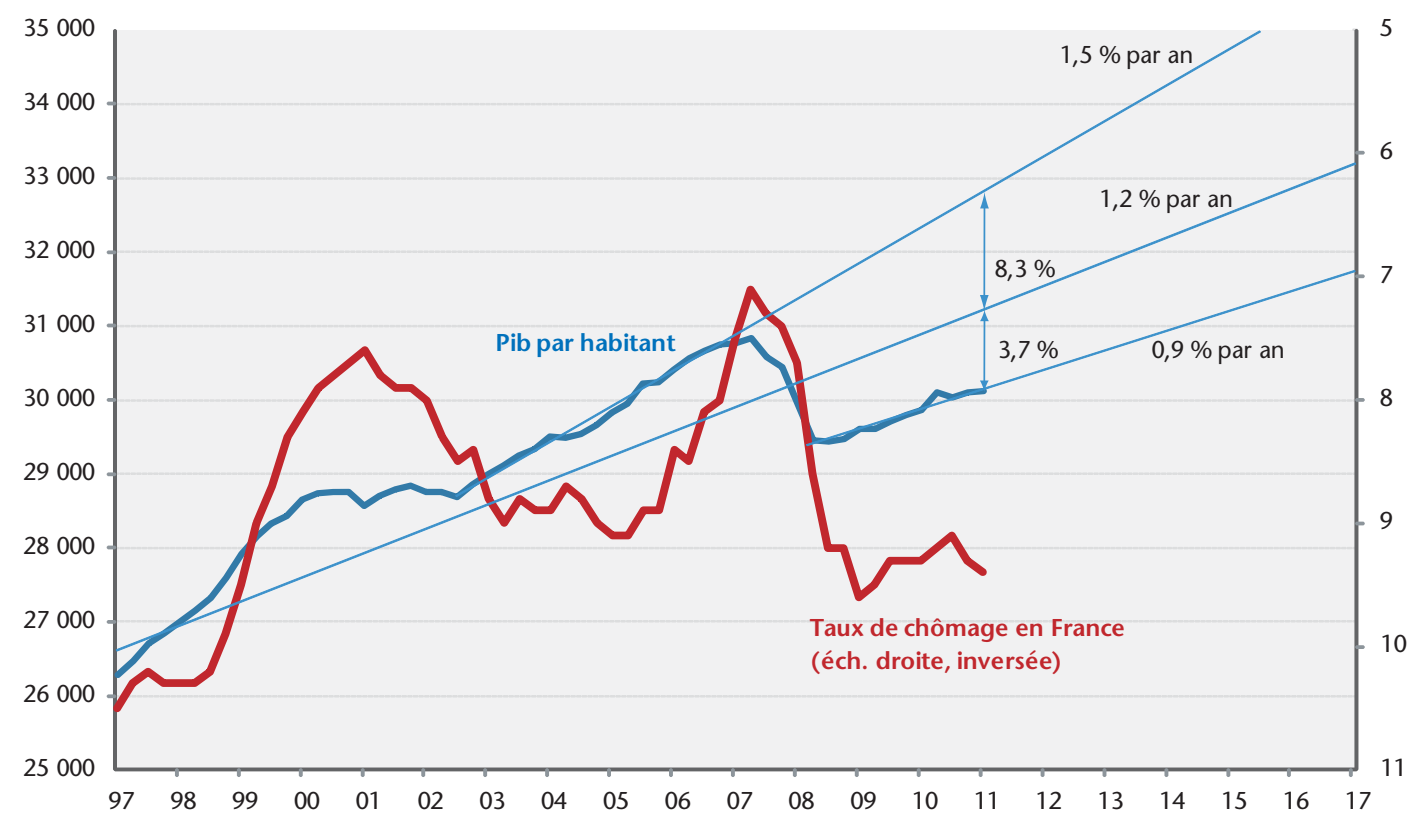

Source : INSEE, calculs et prévisions OFCE, mars 2012. 


\section{Les multiplicateurs budgétaires}

Il existe aujourd'hui un consensus relatif sur la valeur des multiplicateurs budgétaires à court terme (1 à 2 ans). La valeur des multiplicateurs est estimée soit à partir de modèles macroéconomiques, qu'ils soient macroéconométriques (les modèles de l'OFCE) ou DSGE (les modèles des grandes banques centrales) ou à partir d'analyses historiques (comme celle menée par le FMI). On pourra se reporter à Creel, Heyer et Plane (2011) pour une revue de littérature, Coenen et al $(2012)^{2}$ et à Creel $(2012)^{3}$ pour un résumé de l'approche par les DSGE et à Leigh et Pescatori (2011) 4 pour l'approche historique. Au total, on retient un multiplicateur positif, ce qui écarte complètement la possibilité d'une restriction budgétaire expansionniste, proche de l'unité, quoique généralement inférieur à 1 . Il apparaît également que le multiplicateur est d'autant plus grand que l'économie est dans une situation de panique financière, de chômage élevée ou de bas de cycle.

À partir des derniers chiffres de prévisions des grands instituts économiques, nous avons évalué, à partir des informations fournies (croissance effective et potentielle, solde structurel) les multiplicateurs budgétaires implicites à court terme utilisés dans les prévisions pour la France en 2012. Cela suppose notamment de faire une hypothèse de croissance spontanée de l'économie hors effet de l'impact des politiques budgétaires mais qui intègre en revanche les autres chocs, notamment celui du pétrole. La Commission européenne estime un multiplicateur autour de 1 , alors que le MINEFI en donne une évaluation plus faible $(0,7)$. Nous l'évaluons à 1,2 . Cette différence conduit en général les gouvernements à estimer un impact des politiques budgétaires sur l'activité moindre que celui de nos estimations.

Évaluations des multiplicateurs budgétaires implicites pour la France utilisés par les grands organismes de prévision

\begin{tabular}{|c|c|c|}
\hline \multirow{4}{*}{ PIB (en \%, en volume) (1) } & OCDE & 0,3 \\
\hline & Commission européenne & 0,6 \\
\hline & MINEFI*** & 1,0 \\
\hline & OFCE & 0,2 \\
\hline \multirow{4}{*}{ PIB potentiel (en \%, en volume) (2) } & OCDE & 1,5 \\
\hline & Commission européenne & 1,0 \\
\hline & MINEFI*** & 1,7 \\
\hline & OFCE & 1,5 \\
\hline \multirow{4}{*}{ Impulsion budgétaire* (en \% du PIB) (3) } & OCDE & $-1,6$ \\
\hline & Commission européenne & $-0,7$ \\
\hline & MINEFI*** & $-1,5$ \\
\hline & OFCE & $-1,4$ \\
\hline \multirow{4}{*}{ Supplément de croissance $(e n \% d u P I B)^{* *}(4)$} & OCDE & 0,4 \\
\hline & Commission européenne & 0,4 \\
\hline & MINEFI*** & 0,4 \\
\hline & OFCE & 0,4 \\
\hline \multirow{4}{*}{$\begin{array}{l}\text { Croissance spontanée du PIB (en \%, en volume) } \\
(5=2+4)\end{array}$} & OCDE & 1,9 \\
\hline & Commission européenne & 1,4 \\
\hline & MINEFI*** & 2,1 \\
\hline & OFCE & 1,9 \\
\hline \multirow{4}{*}{ Multiplicateur budgétaire implicite (1-5)/(3) } & OCDE & 1,0 \\
\hline & Commission européenne & 1,1 \\
\hline & MINEFI*** & 0,7 \\
\hline & OFCE & 1,2 \\
\hline
\end{tabular}

* Calculé comme l'inverse de la variation du solde structurel

** Fermeture spontanée de l'outputgap hors politique budgétaire mais y compris autres chocs (pétrole, monétaires...)

*** les chiffres correspondent à ceux du Rapport Economique Social et Financier de novembre 2011 (mis à jour après I'annonce du plan de rigueur du 7 novembre 2011).

Sources: Prévisions OFCE mars 2012, Perspectives économiques de l'OCDE de novembre 2011, Prévisions économiques d'Automne 2011 de la Commission Européenne, RESF mis à jour (novembre 2011) du PLF 2012 du MINEFI, calculs OFCE, 


\begin{abstract}
Au-delà du court terme, il existe un consensus discutable sur le fait que les multiplicateurs sont nuls. Ce consensus reflète cependant une hypothèse de construction des modèles plutôt qu'une conséquence d'autres hypothèses ou d'une tentative de mesure à partir de données historiques. Il est cependant difficile d'infirmer ou de confirmer cette hypothèse, pourtant cruciale pour traiter de la question de la soutenabilité de la dette à long terme. La convergence du consensus ne suffit pas en soi à justifier le choix particulier qui est généralement fait d'un multiplicateur nul à long terme. Il existe beaucoup d'arguments pour soutenir que les multiplicateurs sont non nuls à long terme directement ou indirectement (voir de Grauwe (2010) par exemple, les travaux sur l'hystérèse, ou les modèles façon Philippe Aghion sur la R\&D ou les modèles à agents hétérogènes). Dans le cadre de cet exercice de prévision, I'horizon temporel ne nécessite pas de préciser les multiplicateurs à long terme. Leurs valeurs sont d'une grande importance pour la discussion du « new normal » et du risque d'une trajectoire autoréalisée.

1. Creel, J., Heyer, É., \& Plane, M. (2011), «Petit précis de politique budgétaire par tous les temps Les multiplicateurs budgétaires au cours du cycle », Revue de l'OFCE, n 116 / janvier 2011.

2. Coenen, G., Christopher J. Erceg, Freedman, C., Furceri, D., Kumhof, M., Lalonde, R., Laxton, D., et al. (2012),

«Effects of fiscal stimulus in structural models», American Economic Journal: Macroeconomics, 4(1), 22-68,

International monetary fund (IMF).

3. http://www. ofce.sciences-po.fr/blog/?p=1363

4. Leigh, D., \& Pescatori, A. (2011), «Expansionary Austerity New International Evidence », IMF Working Paper, $(11 / 158)$.
\end{abstract}

\title{
Politique monétaire : assouplissement consensuel
}

La dégradation des perspectives économiques et le maintien du chômage à un niveau élevé a poussé les principales banques centrales (BCE, FED et Banque d'Angleterre) à instaurer de nouvelles mesures d'assouplissement fin 2011 et début 2012, malgré le regain de tensions inflationnistes liées à la forte poussée des prix du pétrole fin 2011-début 2012. En particulier, la dégradation des conditions de financement en zone euro ont conduit la BCE à infléchir sa politique et à injecter de la liquidité dans le secteur bancaire. Alors que tous les pays développés sont, à des degrés divers, engagés dans un processus de réduction des déficits, l'outil monétaire est donc le seul capable de limiter l'effet récessif sur les économies développées, d'où l'utilisation expansionniste qui en est faite par les autorités monétaires.

Alors que la BCE avait manifesté sa volonté de normaliser sa politique monétaire en remontant ses taux mi-2011, et malgré une progression de l'inflation en 2011 (2,7\%), la morosité du marché du crédit en zone euro l'a conduite à infléchir sa politique en abaissant fin 2011 son taux directeur pour le ramener à $1 \%$. Pour soutenir le renforcement de l'activité de prêts bancaires et de la liquidité du marché monétaire de la zone euro, la BCE a également décidé de mener deux opérations de refinancement à long terme (LTRO) à échéance de 3 ans : la première, qui a eu lieu le 21 décembre 2011, a permis d'octroyer 489 milliards aux banques au taux de $1 \%$; la seconde du 29 février 2012 a alloué 529,5 milliards d'euros à 800 banques européennes, toujours à trois ans et toujours à $1 \%$, ce qui a favorisé leur retour sur le marché des titres obligataires privés. En outre, depuis mai 2011, la BCE mène une politique d'achat de dette publique des pays en crise (pour un montant estimé à 219 milliards d'euros début mars 2012). Au total, ces opérations ont conduit à une forte augmentation de son actif, passé de 2000 milliards d'euros fin 2011 à 3000 milliards en mars 2012. Étant donnée le rôle plus important du crédit bancaire dans la zone euro qu'aux États-Unis, la BCE reste particulièrement attentive à l'évolution des conditions de crédit, même si la 
progression du chômage en zone euro et la récession dans certains pays (Portugal, Grèce, Italie) ont dû également motiver ses décisions.

Le bilan de la Réserve fédérale américaine, gonflé par les mesures d'assouplissement quantitatif QE1 et QE2, a moins progressé, passant de 2600 milliards de dollars mi2011 à 2800 milliards en mars 2012. La Réserve fédérale américaine a décidé de laisser ses taux directeurs inchangés au vu de la situation de l'emploi toujours dégradée. En affichant ses prévisions à I'horizon fin 2014, la Réserve fédérale prévient que le taux de chômage restera probablement au-dessus du niveau normal de long terme estimé à $6 \%$. Dans ces conditions, et pour soutenir la croissance, elle maintient le taux objectif des Fonds fédéraux à leur niveau plancher et confirme que compte tenu du niveau de croissance, sa politique restera très accommodante à I'horizon fin 2014. Même si un troisième plan d'assouplissement quantitatif (QE 3) semble peu probable à court terme, en raison de la résurgence de l'inflation, il pourrait néanmoins intervenir courant 2012 si les perspectives se dégradent sur le plan de l'emploi : la Fed pourrait ainsi acquérir soit des obligations d'État, soit des créances immobilières à long terme, avec pour objectif principal de soutenir le marché immobilier américain. Ces liquidités seraient toutefois «stérilisées », ce qui permettrait de maintenir l'inflation à des niveaux bas.

La situation est identique au Royaume-Uni où la Banque d'Angleterre s'inquiète davantage de la détérioration de la situation économique que de l'accélération de l'inflation. Le Comité de politique monétaire (CPM) de la Banque d'Angleterre a massivement soutenu la croissance depuis le début de la crise, laissant de côté l'objectif d'inflation qui lui est donné par le gouvernement. Le CPM a en effet pour mandat de tout mettre en œuvre pour que l'inflation soit proche de $2 \%$ (en termes d'IPCH), dans une fourchette de +/-1\%. Or I'inflation a franchi $3 \%$ à l'automne 2010, pour accélérer ensuite et atteindre 5,2 \% en août 2011. Mais le CPM a laissé son taux directeur inchangé à 0,5\% depuis mars 2009, et a par deux fois augmenté son programme d'achats d'actifs depuis l'automne dernier, le portant de 200 à 275 milliards de livres en octobre 2011, puis à 300 milliards de livres en février 2012. Le CPM avait justifié l'absence de durcissement de sa politique par le caractère temporaire de l'accélération de l'inflation (du fait de deux hausses successives de TVA en janvier 2010 et janvier 2011 et de la hausse des prix des matières premières), alors que l'économie est en sous-emploi. Au début du printemps 2012, tous les membres étaient favorables à un statu quo monétaire, à l'exception de deux membres (David Miles et Adam Posen), ayant voté en faveur d'une hausse de 25 milliards du programme des achats d'actifs. Les perspectives de poursuite de ralentissement de I'inflation à I'horizon de la fin 2013, avec un retour de l'inflation à $2 \%$ permettront au CPM de poursuivre une politique de soutien à la croissance.

\section{Le retour de la récession en zone euro}

Le nouvel affaissement de la croissance en zone euro, que laissait craindre le retournement des indicateurs conjoncturels à l'été 2011 (graphique 2), s'est matérialisé dans la seconde moitié de l'année, avec une croissance très faible au troisième trimestre, $+0,1 \%$, et un franc recul de l'activité au quatrième, $-0,3 \%$. Par contraste, les ÉtatsUnis, après un ralentissement au premier semestre $2011(+0,4 \%$ sur le semestre), ont rebondi dans la seconde moitié de l'année $(+1,2 \%)$ et la croissance japonaise s'est relevée du traumatisme provoqué par les catastrophes naturelles au premier trimestre grâce à la mise en place d'une politique budgétaire vigoureuse (recul du PIB de 2,1\% au premier semestre, contre une hausse de 1,5\% au deuxième). 
Ces séquences de ralentissements en cours d'année ont affecté négativement la croissance des différentes zones en moyenne annuelle (tableau 1), de $3 \%$ en 2010 à 1,7\% en 2011 aux États-Unis, de 1,8 à 1,5\% en zone euro, de 4,7\% à -0,6\% au Japon. Les pays émergents n'ont pas constitué un soutien à la croissance mondiale aussi dynamique qu'en 2010, avec un recul de la croissance de 9,6 à 7,3 \% en Asie, de 6,2 à 4,3\% en Amérique Latine. Seuls les pays de l'Est ont échappé au ralentissement avec une hausse du PIB de la zone de $4,1 \%$ en 2011, contre $3,6 \%$ en 2010, mais la reprise après la récession y a été différée et ces pays n'ont pas échappé à un net coup de frein en fin d'année 2011.

Graphique 2. PIB par tête en parité de pouvoir d'achat*
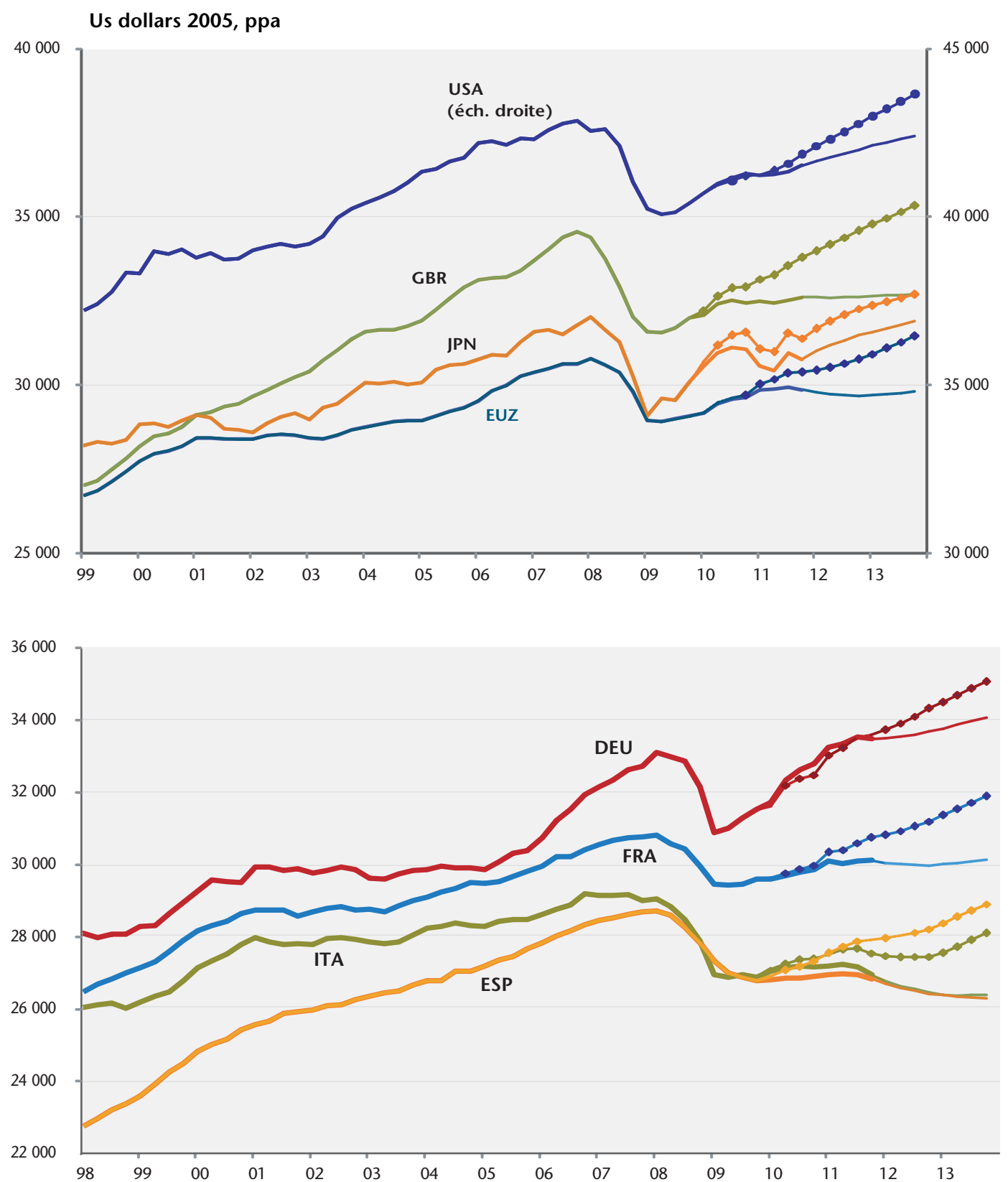

* En pointillés, la trajectoire spontanée des PIB par tête sans l'effet des politiques budgétaires restrictives.

Sources : Comptabilités nationales, calculs OFCE. 
Au sein des pays de la zone euro, le ralentissement est généralisé, sauf dans quelques économies qui se distinguent, avec une accélération de la croissance en moyenne annuelle en France et en Espagne notamment. Ces résultats sont toutefois en trompe-I'œil. L'économie espagnole est restée très en retrait des autres grandes économies depuis la reprise de 2009 , et n'a d'ailleurs pas échappé à un recul de son PIB au deuxième semestre 2011. Les résultats français au quatrième trimestre 2011 ont surpris les observateurs, avec une croissance de $+0,2 \%$, quand simultanément quasiment tous les pays de la zone euro affichent un recul de leur PIB. Toutefois, le «mystère français » sera peut-être levé lors des révisions ultérieures des comptes nationaux, dont une étude récente a mis en évidence le caractère pro-cyclique en période de retournement conjoncturel à la baisse, phase à laquelle s'apparente la situation de l'économie française en 2011 ; les comptes nationaux préliminaires tendent à surestimer la croissance et sont par conséquent révisés en baisse jusqu'à leur version définitive.

Les économies développées portent toujours les stigmates de la crise financière, aucun des pays, hormis l'Allemagne, n'ayant rattrapé son niveau d'activité d'avantcrise (graphique 1). En termes de PIB par tête, les pays sont toujours en récession, les économies anglo-saxonnes restant les plus marquées avec celles de l'Europe du Sud, I'Italie et I'Espagne (tableau 2). La France et le Japon figurent en position intermédiaire, la première grâce à une chute d'activité en 2008-2009 moindre qu'ailleurs, et le second grâce à un rebond plus marqué jusqu'à la mi-2010. Seule l'Allemagne émerge en ayant profité pleinement de la reprise de commerce mondial.

\section{Tableau 2. Évolution des PIB par tête et du chômage}

\begin{tabular}{lccccccc} 
& USA & JPN & GBR & DEU & FRA & ITA & ESP \\
\hline Pib/tête1 & $-0,8 \%$ & $-0,4 \%$ & $-0,8 \%$ & $+0,4 \%$ & $-0,2 \%$ & $-1,4 \%$ & $-0,8 \%$ \\
Taux de chômage $^{2}$ & $+3,9$ & $+0,5$ & $+2,5$ & $-1,1$ & $+2,3$ & $+2,2$ & $+13,6$ \\
\hline
\end{tabular}

1 : Taux de croissance annualisé moyen du PIB réel par tête depuis le point haut du quatrième trimestre 2007 (États-Unis et Royaume-Uni) ou du premier trimestre 2008 pour les autres pay jusqu'au 4e trimestre 2011.

2 : Variation du chômage en point sur la même période.

Sources : Comptabilités nationales, calculs OFCE.

Sur le front du chômage, le bilan de près de quatre années de récession n'est guère reluisant. Les pays font face à la persistance du sous-emploi, à des degrés qui toutefois ne reflètent pas nécessairement l'intensité des pertes de production. Le gonflement du chômage a été important aux États-Unis, au Royaume-Uni et en Espagne, du fait d'une plus grande flexibilité des marchés du travail et d'un impact de la crise sur la construction, secteur intensif en main-d'œuvre, plus prononcé. Le chômage n'a pas augmenté en Allemagne, et moins qu'ailleurs au Japon, d'abord parce que le recours massif au chômage partiel a maintenu les licenciés potentiels dans l'emploi pendant la crise de 2008-09, ensuite parce que l'ouverture des ces économies au commerce mondial leur a permis de bénéficier de la réactivation des échanges, enfin parce que la population active y est moins dynamique.

Le retournement conjoncturel qui s'est amorcé à la mi-2011 dans la zone euro s'est donc effectué dans la plupart des pays en partant d'un niveau de chômage élevé et est susceptible de fragiliser de nouveaux ménages en plus de ceux déjà frappés (graphique 3). Les perspectives de retour à l'emploi s'éloignant, le risque de tomber dans le chômage de longue durée s'accroît significativement avec à la clé un appauvris- 
sement supplémentaire des ménages en passe de sortir des dispositifs d'indemnisation pour basculer dans les minima sociaux.

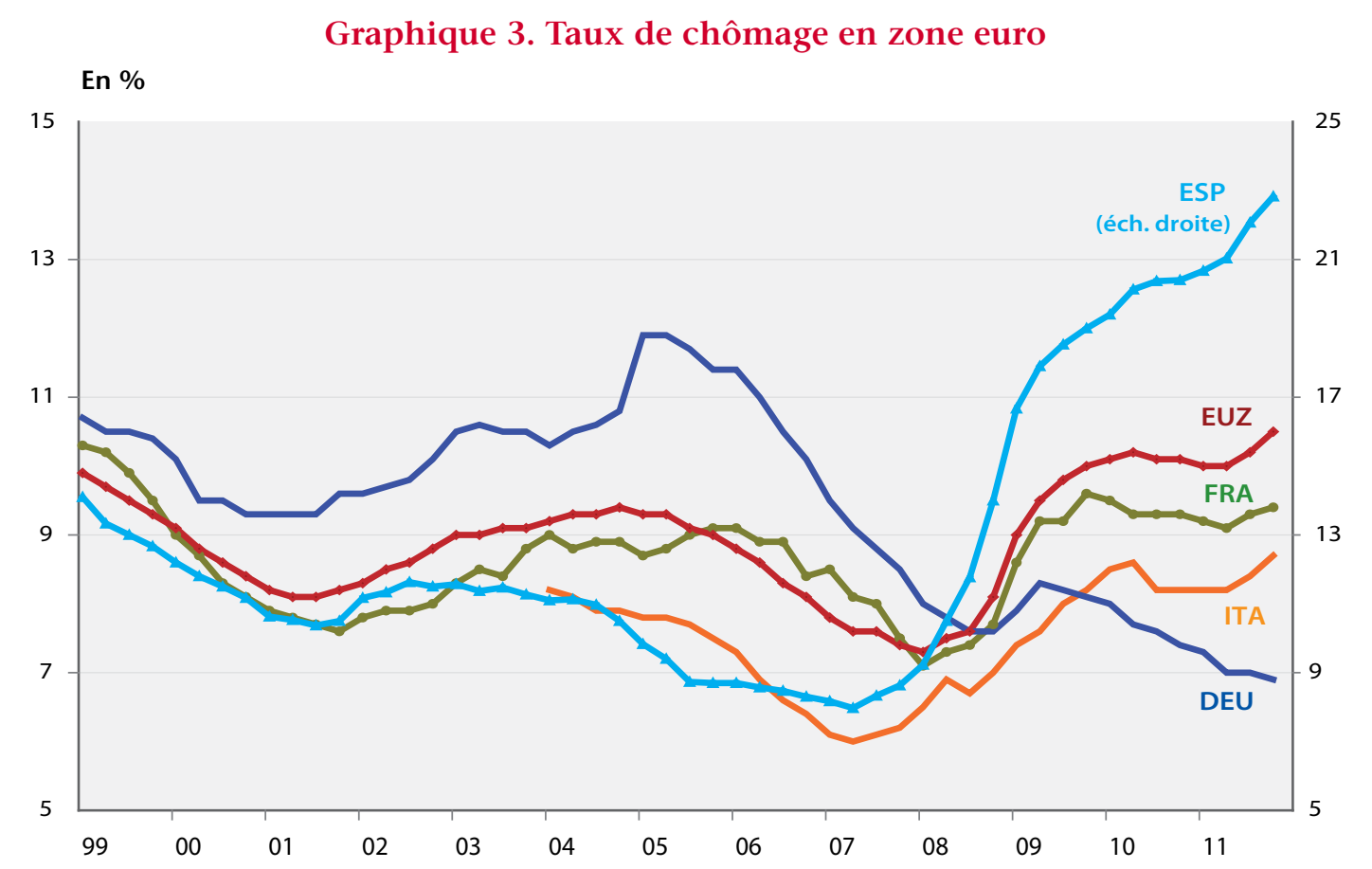

Source : Eurostat.

L'extension du chômage de longue durée est source d'amplification des impulsions négatives subies par l'économie de la zone euro. La montée du chômage stimule l'épargne de précaution face au risque grandissant de perte d'emploi ressenti par les ménages, réduit davantage le pouvoir d'achat de ceux qui sont tombés dans le chômage de longue durée, et ce dans un contexte de résurgence de l'inflation des produits énergétiques. Les effets des politiques d'assainissement budgétaire mises en œuvre en Europe en 2011 et en 2012 risquent alors d'être amplifiés, d'abord parce que les multiplicateurs sont plus élevés en bas de cycle, comme actuellement du fait de la persistance du chômage, ensuite parce qu'elles ne sont pas coordonnées mais appliquées simultanément sans précaution.

\section{Le découplage? Pas vraiment...}

Après un troisième trimestre assez encourageant dans les pays avancés, marqué par un redressement très dynamique au Japon après Fukushima $(+1,7 \%)$, et une progression soutenue de la consommation et de l'investissement aux États-Unis (conduisant à une croissance de 0,5\%), le dernier trimestre 2011 dresse un tableau beaucoup moins favorable. Alors qu'on attendait une poursuite de la reprise japonaise, compte tenu de l'effort de relance, la situation conjoncturelle a fortement dégradé la balance commerciale du Japon (rupture des chaînes d'approvisionnement à la suite des inondations en Thaillande et importations d'énergie en hausse), conduisant à une baisse du PIB. En zone euro, après deux trimestres de croissance molle, le PIB a reculé sous I'effet de l'impulsion budgétaire négative généralisée, chiffrée à 1,1 point de PIB en 2011, qui a pesé principalement sur la consommation des ménages et des administra- 
tions, plongeant cinq pays (Italie, Pays-Bas, Belgique, Portugal et Grèce) dans la récession. Seuls les États-Unis affichent une croissance soutenue, terminant l'année 2011 à un rythme annualisé de $3 \%$, grâce à plusieurs facteurs : la décrue du taux d'épargne, l'augmentation du revenu disponible, la baisse du chômage, et la reconstitution des marges des entreprises.

Les économies émergentes et en développement ont subi l'impact de cette piètre performance des pays développés au dernier trimestre 2011, surtout via le canal du commerce international et le canal financier. En 2012 et 2013, la croissance des économies émergentes pâtirait de la détérioration de l'environnement extérieur ; le maintien d'une croissance soutenue dépendra donc de la capacité de la demande intérieure à prendre le relais de la croissance.

La crise souveraine qui frappe l'Europe a contaminé les marchés actions et obligations et provoqué une baisse des flux de capitaux vers les marchés émergents. Pour pallier cette situation, les pays émergents ont poursuivi le resserrement monétaire, ce qui a entraîné une inflexion de la croissance au dernier trimestre 2011. Les PECO ont enregistré de piètres performances au dernier trimestre 2011. Non seulement les pays les plus exposés au commerce avec la zone euro (comme la République tchèque) ont été atteints de plein fouet, mais en outre, la demande interne n'a pas pu jouer le rôle d'amortisseur. En effet, comme dans les autres pays européens, la plupart des PECO ont mis en œuvre des politiques d'austérité budgétaire se traduisant par une baisse généralisée de la demande publique et par une stagnation de la consommation privée. La situation est meilleure en Asie et en Inde : certes, le commerce extérieur a contribué négativement à la croissance, mais ceci a été compensé par la croissance de la demande intérieure. La croissance en Amérique latine est restée très soutenue au dernier trimestre 2011, la région étant moins directement exposée au choc venant de la zone euro; elle a de plus bénéficié du dynamisme de la croissance nord-américaine.

Le risque existe que la crise se propage en outre par le canal financier. Le ralentissement, voire l'inversion des flux d'IDE et d'investissements de portefeuille, fragiliserait les économies émergentes, en particulier celles qui présentent un besoin de financement extérieur. Un recul marqué des flux de capitaux vers les pays émergents pourrait entraîner des ajustements de la demande interne pour les pays ayant des déficits courants, comme c'est notamment le cas en Amérique latine (Brésil, Pérou, Mexique). Les PECO (notamment la Pologne, la Hongrie, la République tchèque ou la Roumanie) sont également très exposés à ce risque étant donné le montant des IDE réalisés par les banques européennes et américaines.

Sous l'effet de la purge budgétaire en zone euro, la croissance mondiale ralentirait en 2012. La tendance baissière sur les matières premières se poursuivrait en 2012, sous l'effet du ralentissement de la demande mondiale. Les tensions sur les prix devraient donc s'atténuer, ce qui permettrait à certains pays de retrouver une politique monétaire plus accommodante. Les pays d'Europe centrale et orientale seront les plus touchés par la restriction budgétaire généralisée, étant donné leurs liens économiques et commerciaux avec les pays de la zone euro et la faiblesse persistante de leur demande intérieure. Ainsi, les nouveaux pays membres de l'Union européenne verraient leur croissance divisée par plus de deux, passant de 3,1\% en 2011 à 1,3\% en 2012. Les anciens pays de la CEI pâtiraient également de l'atonie en zone euro, même si le dynamisme de la Russie, lié au niveau élevé des prix du pétrole, atténuera l'impact dépressif de la baisse de la demande de la part des pays européens. Globale- 
ment, l'Asie resterait la zone la plus dynamique, affichant une croissance qui atteindrait $7,0 \%$ en 2012 et 7,2 \% en 2013. Cependant, on s'attend à un découplage au sein des pays asiatiques. La croissance dans les pays d'Asie les plus fortement dépendants des exportations et ne disposant pas d'un marché intérieur suffisant, comme Singapour, Taiwan, la Thaïlande, la Malaisie et les Philippines, baisserait en rythme annuel en 2012 et 2013. En revanche, en Chine, en Inde ou en Corée du Sud, la demande intérieure devrait prendre le relais, favorisée par une politique monétaire plus accommodante, le risque d'une surchauffe inflationniste ayant été écarté du fait de la faiblesse de la demande mondiale et de la baisse des niveaux de prix hors énergie. En conséquence de la contraction du commerce extérieur, on s'attend d'ailleurs à une contribution nulle de la balance commerciale à la croissance chinoise en 2012 et 2013. En Chine, la croissance resterait forte, mais serait en légère baisse, avec 8,3\% en 2012 et 8,1 \% en 2013, contre $9,2 \%$ en 2011. En Amérique latine, la croissance ralentirait, passant de 4,3\% en 2011 à 3,3\% en 2012.

\section{La restriction budgétaire des uns ...}

La réponse de l'économie à une politique de rigueur budgétaire dépend de sa position dans le cycle ${ }^{4}$ et de l'efficacité de la politique monétaire. En mettant en œuvre des politiques d'assainissement budgétaire en période de creux conjoncturel alors que le chômage reste élevé et que la politique monétaire est défaillante, les gouvernements européens ont interrompu le processus de reprise lente de l'économie, et ils ont pris le risque de faire replonger l'économie de la zone euro dans la récession et de favoriser une nouvelle montée du chômage.

Les impulsions budgétaires seront en effet partout négatives en 2012 et en 2013, tant en zone euro que dans les pays anglo-saxons ainsi que dans les pays de l'Est (tableau 3). Sans surprise, sur les années 2012 et 2013, les restrictions budgétaires seront les plus intenses dans les pays de la zone euro où les difficultés budgétaires sont les plus criantes, les pays du Sud de l'Europe, avec des impulsions négatives approchant ou dépassant 5 points de PIB sur les deux années, mais aussi I'Irlande. À un degré moindre, les autres pays de la zone contribueront aussi à la restriction, avec des impulsions comprises entre $-3,1$ points de PIB pour la France et $-1,7$ pour I'Autriche. À nouveau l'Allemagne se distinguera avec une impulsion quasi-neutre, justifiée par une situation budgétaire plus soutenable qu'ailleurs après le fort rebond de la croissance depuis 2009 qui a amélioré significativement la composante conjoncturelle du déficit. Le reste du monde développé mènera également des politiques restrictives, tout comme les PECO, hormis le Japon qui continuera à soutenir son activité pour parer aux conséquences négatives des catastrophes de mars 2011.

4. Voir Creel J., É. Heyer et M. Plane, 2011, «Petit précis de politique budgétaire par tous les temps: les multiplicateurs budgétaires au cours du cycle », Revue de l'OFCE, n 116, janvier. 
Qui sème la restriction récolte la récession : Perspectives économiques 2012-2013

Tableau 3. Impulsions et déficits budgétaires

\begin{tabular}{|c|c|c|c|c|c|c|c|c|c|}
\hline \multicolumn{10}{|c|}{$\begin{array}{l}\text { En points de PIB } \\
\text { Impulsions budgétaires }\end{array}$} \\
\hline & 2012 & 2013 & $\begin{array}{c}2012 / \\
2013\end{array}$ & 2011 & 2012 & 2013 & 2011 & 2012 & 2013 \\
\hline GRC & $-5,3$ & $-4,3$ & $-9,6$ & $-9,3$ & $-7,3$ & $-4,6$ & 161 & 136 & 139 \\
\hline ESP & $-3,4$ & $-2,9$ & $-6,3$ & $-8,5$ & $-6,5$ & $-4,5$ & 68 & 75 & 80 \\
\hline IRL & $-3,0$ & $-2,6$ & $-5,6$ & $-10,1$ & $-8,7$ & $-7,5$ & 110 & 119 & 124 \\
\hline PRT & $-3,0$ & $-2,1$ & $-5,1$ & $-4,0$ & $-4,5$ & $-3,5$ & 99 & 106 & 109 \\
\hline ITA & $-2,9$ & $-1,7$ & $-4,6$ & $-3,8$ & $-2,8$ & $-1,7$ & 120 & 123 & 124 \\
\hline FRA & $-1,6$ & $-1,5$ & $-3,1$ & $-5,2$ & $-4,4$ & $-3,5$ & 86 & 89 & 90 \\
\hline NLD & $-1,9$ & $-1,0$ & $-2,9$ & $-5,0$ & $-4,5$ & $-4,0$ & 66 & 70 & 73 \\
\hline FIN & $-1,1$ & $-1,3$ & $-2,4$ & $-1,2$ & $-0,9$ & $-0,6$ & 47 & 46 & 45 \\
\hline BEL & $-1,4$ & $-0,8$ & $-2,2$ & $-4,0$ & $-3,4$ & $-3,0$ & 96 & 97 & 98 \\
\hline AUT & $-1,2$ & $-0,5$ & $-1,7$ & $-3,4$ & $-3,0$ & $-3,0$ & 72 & 73 & 75 \\
\hline DEU & $-0,3$ & $-0,1$ & $-0,4$ & $-1,0$ & $-1,1$ & $-1,3$ & 80 & 79 & 79 \\
\hline PECO & $-1,7$ & $-1,7$ & $-3,4$ & - & - & 2,4 & - & - & - \\
\hline GBR & $-2,0$ & $-1,5$ & $-3,5$ & $-8,3$ & $-7,5$ & $-6,9$ & 86 & 92 & 97 \\
\hline USA & $-1,1$ & $-1,5$ & $-2,6$ & $-9,7$ & $-8,6$ & $-7,1$ & 102 & 107 & 110 \\
\hline PJN & 0,4 & 0,5 & 0,9 & $-9,3$ & $-9,2$ & $-9,8$ & 212 & 219 & 228 \\
\hline
\end{tabular}

Sources : Projets de lois de finance, calculs et prévisions OFCE.

Ces politiques budgétaires restrictives auront des effets négatifs sur l'activité d'autant plus forts qu'elles sont menées dans une situation conjoncturelle où les écarts de production négatifs restent importants. En déprimant l'activité, ces politiques ne produiront pas une amélioration des finances publiques à la hauteur de l'effort consenti ex ante. Leur conséquence sera de réactiver le jeu des stabilisateurs automatiques en générant des pertes de recettes fiscales et en occasionnant une nouvelle vague de dépenses sociales à destination des ménages fragilisés par le freinage de l'activité. S'y ajoutera la résistance à la baisse des ratios de dette publique et de déficit liée à une progression du PIB inférieure aux attentes.

\section{... l'affaire de tous}

En économie ouverte, les chocs négatifs générés par les politiques budgétaires restrictives ne restent pas cantonnés aux seules économies nationales mais se répercutent sur l'activité des partenaires commerciaux. La restriction budgétaire d'un pays se transmet aux autres par le canal du commerce international : le freinage des importations, lié au ralentissement de l'activité dû au resserrement budgétaire, induit aussi un freinage des exportations chez ses fournisseurs. Si dans le même temps les fournisseurs eux-mêmes mènent des politiques restrictives, ils subissent, en plus du choc extérieur, leur propre choc négatif et le transmettent en retour à leurs partenaires, de telle sorte 
que des politiques d'assainissement budgétaire non coordonnées entre en résonance et démultiplient leurs effets.

Les croissances spontanées, qui varient selon les pays mais qui sont toutes largement positives en 2012 et en 2013, seront donc entravées par ce double effet des politiques restrictives (tableau 4). Même le Japon, qui aurait pu prétendre à une croissance effective supérieure à $2 \%$ en 2012 et proche de ce seuil en 2013, verra l'impact de ses impulsions budgétaires positives annulé cette année et fortement rogné en 2013 par la restriction de ses partenaires commerciaux.

Tableau 4. Effets directs et indirects des politiques budgétaires restrictives

En points de PIB

\begin{tabular}{|c|c|c|c|c|c|c|}
\hline & & ... effet direct & $\begin{array}{c}\text {... effet via } \\
\text { la demande } \\
\text { adressée }\end{array}$ & Effet total & $\begin{array}{c}\text { PIB } \\
\text { prévu }\end{array}$ & $\begin{array}{c}\text { PIB hors } \\
\text { politiques } \\
\text { budgétaires }\end{array}$ \\
\hline \multirow{2}{*}{ FRA } & 2012 & $-1,4$ & $-0,9$ & $-2,3$ & 0,2 & 2,5 \\
\hline & 2013 & $-1,2$ & $-0,7$ & $-1,9$ & 0,7 & 2,6 \\
\hline \multirow{2}{*}{ DEU } & 2012 & $-0,3$ & $-1,1$ & $-1,4$ & 0,3 & 1,7 \\
\hline & 2013 & $-0,1$ & $-1,0$ & $-1,1$ & 0,8 & 1,9 \\
\hline \multirow{2}{*}{ ESP } & 2012 & $-3,1$ & $-0,9$ & $-3,9$ & $-1,1$ & 2,8 \\
\hline & 2013 & $-2,6$ & $-0,7$ & $-3,3$ & $-0,6$ & 2,7 \\
\hline \multirow{2}{*}{ ITA } & 2012 & $-2,0$ & $-0,9$ & $-2,9$ & $-1,7$ & 1,2 \\
\hline & 2013 & $-1,2$ & $-0,7$ & $-1,9$ & $-0,9$ & 1,0 \\
\hline \multirow{2}{*}{ GBR } & 2012 & $-1,8$ & $-0,7$ & $-2,5$ & 0,7 & 3,2 \\
\hline & 2013 & $-1,3$ & $-0,6$ & $-1,9$ & 0,9 & 2,8 \\
\hline \multirow{2}{*}{ USA } & 2012 & $-0,8$ & $-0,3$ & $-1,1$ & 2,3 & 3,4 \\
\hline & 2013 & $-1,1$ & $-0,2$ & $-1,4$ & 2,4 & 3,8 \\
\hline \multirow{2}{*}{ JPN } & 2012 & 0,4 & $-0,4$ & 0,0 & 1,9 & 1,9 \\
\hline & 2013 & 0,5 & $-0,3$ & 0,2 & 1,5 & 1,3 \\
\hline
\end{tabular}

Sources : Comptabilités nationales, Projets de lois de finance, calculs et prévisions OFCE.

\section{L'étau des financements}

Les engagements des gouvernements en matière de réduction des déficits publics sont établis en référence à une trajectoire anticipée. Or un ralentissement de l'activité plus fort que celui sur lequel sont bâtis les budgets pour 2012 et 2013 conduirait à un creusement plus marqué des déficits conjoncturels susceptible de contrebalancer les efforts ex-ante consentis par les États et aboutirait au final à un non-respect des engagements initiaux.

Ce pourrait être le cas pour l'Italie et l'Espagne au cours des deux prochaines années, et pour l'Allemagne et la France en 2013. Il faudrait alors s'attendre à de nouveaux tours de vis budgétaires si les gouvernements tenaient, quoi qu'il advienne et quel que soit l'état de la conjoncture, à respecter leurs engagements. La retombée en récession de la zone euro deviendrait alors inéluctable, et elle serait d'autant plus violente que les nouvelles restrictions seraient encore une fois synchrones ${ }^{5}$. 
Ces conjectures illustrent bien le problème auquel sont confrontés les pays de la zone euro. Les doutes sur la solvabilité des pays, quelle que soit leur notation à l'exception de l'Allemagne, rendent plus difficile le financement des déficits et de la fraction de la dette qui devra être renouvelée cette année et l'année prochaine. Pour assurer les marchés de leur pleine capacité à honorer leur dette et éviter des hausses de taux qui les étrangleraient davantage, les gouvernements conduisent des politiques restrictives qui étouffent toute capacité de rebond spontané de leurs économies et toute perspective de résorption des déficits conjoncturels. En lieu et place de rebond, c'est au mieux la stagnation qui prévaut, repoussant toute perspective de levée des sanctions.

\section{Trois scénarios sous haute tension}

Notre prévision décrit un scénario mondial dans lequel les économies industrialisées enregistrent un ralentissement marqué de la croissance du PIB, voire une récession pour certains pays de la zone euro, en 2012 et 2013. Dans ce scénario l'output gap se creuse et le chômage monte encore dans la plupart des pays. Nous supposons néanmoins que, hormis les effets négatifs sur la croissance des politiques budgétaires menées simultanément et des prix du pétrole élevés, l'économie mondiale résiste à une nouvelle crise financière malgré la persistance des déséquilibres et un risque social élevé. Ce scénario sous haute tension, dans lequel l'économie mondiale plie mais ne rompt pas, est notre cadre de référence et nous semble le plus probable. Mais, nous avons aussi étudiés trois scénarios à risque dans lesquels une crise de grande ampleur finirait par se produire.

\section{Scénario 1 : la surenchère budgétaire dans la zone euro}

Le scénario à risque le plus probable est celui d'une surenchère budgétaire dans la zone euro, liée à un rétablissement plus lent que prévu des comptes publics associé à une hausse des primes des risques sur dettes souveraines de certains pays.

Les gouvernements européens qui se sont fixés des objectifs ambitieux en matière de réduction de déficit public, notamment en zone euro dans le cadre des programmes de stabilité, se trouveraient confrontés à une dégradation plus forte que prévu de la composante conjoncturelle du solde public. En sous-estimant la valeur des multiplicateurs budgétaires qui sont très élevés à court et moyen termes dans le contexte économique actuel6, les prévisions de croissance pour 2012 établies par les gouvernements pour le cadrage budgétaire des lois de finances deviendraient rapidement obsolètes, révélant la sous-calibration des plans d'austérité budgétaire permettant d'atteindre les objectifs de déficits publics. Cette sous-estimation de l'impact des politiques budgétaires sur la croissance conduirait soit à des nouveaux tours de vis budgétaires accélérant le processus récessif, soit à des déficits publics plus élevés que prévu. La course à la réduction des déficits publics, indépendamment de toute considération conjoncturelle, pèserait sur la croissance et mettrait à mal la soute-

5. Voir la partie « zone euro » de cette chronique.

6. Pour plus de détails, voir Creel et alii (2010) «Multiplicateurs budgétaires au cours du cycle : petit précis de politique budgétaire par tous les temps. », Revue de l'OFCE, n 119, octobre 2011 et IMF (2010), Chapitre 3 « Will It Hurt? Macroeconomic Effects of Fiscal Consolidation », octobre. 
nabilité des finances publiques. La sous-évaluation de la valeur du multiplicateur budgétaire aurait deux conséquences : premièrement les pays, en minimisant les effets de la politique budgétaire sur la composante conjoncturelle du solde public, n'atteindraient pas leurs objectifs de réduction de déficit public inscrits dans les programmes de stabilité ce qui provoquerait la défiance des marchés. Deuxièmement, les politiques budgétaires, en affectant la croissance plus sévèrement que ce qui était prévu par les gouvernements, entraînerait de nombreux pays de la zone euro dans une récession profonde, ce qui renforcerait la crainte des marchés quant à la soutenabilité des finances publiques.

Nous avons simulé un scénario dans lequel les gouvernements tiennent coûte que coûte, et quelle que soit la conjoncture, à respecter leurs engagements budgétaires (tableau 5). Si tel était le cas, cela nécessiterait l'adoption de nouveaux plans restriction budgétaire dans les mois à venir.

Plusieurs cas de figure ont été étudiés. Ils reposent tous sur l'hypothèse d'un multiplicateur budgétaire national de 0,9 correspondant à un multiplicateur total de 1,3. En effet, comme nous l'avons présenté en détail dans un travail récent (OFCE, 20117), la mise en place en période de basse conjoncture de politiques de restriction budgétaire simultanées dans l'ensemble des pays européens et dans une situation de faible marge de manœuvre de la politique monétaire, conduit à une valeur de multiplicateur supérieure à l'unité.

Tableau 5. Soldes publics en 2012 et 2013 prévus par...

En points de PIB

\begin{tabular}{lcccccccc} 
& \multicolumn{2}{c}{ DEU } & \multicolumn{2}{c}{ FR } & \multicolumn{2}{c}{ ITA } & \multicolumn{2}{c}{ ESP } \\
& 2012 & 2013 & 2012 & 2013 & 2012 & 2013 & 2012 & 2013 \\
\hline ... I'OFCE & $-1,1$ & $-1,3$ & $-4,4$ & $-3,5$ & $-2,7$ & $-1,6$ & $-6,5$ & $-4,5$ \\
... les gouvernements & $-1,0$ & $-0,5$ & $-4,5$ & $-3,0$ & $-1,6$ & $-0,1$ & $-5,3$ & $-3,0$ \\
\hline Écart & 0,1 & 0,7 & $-0,1$ & 0,5 & 1,1 & 1,5 & 1,2 & 1,5 \\
\hline
\end{tabular}

Sources : sources nationales, prévisions OFCE.

De manière à isoler l'impact sur la croissance du plan d'économie national et de ceux des partenaires, nous avons supposé que chaque pays respecte, seul, son engagement (tableau 6). Sous cette hypothèse, l'effort supplémentaire à fournir serait considérable en Italie et en Espagne; il représenterait plus de 2 points de PIB en 2012 (respectivement 32,0 et 22,5 milliards d'euros), et près de 1 point de PIB en 2013 (respectivement 13,0 et 10,8 milliards d'euros). La France et l'Allemagne devraient également mettre en œuvre un plan de restriction budgétaire, même s'il est de moindre ampleur, compris entre 0,9 et 1,4 point de PIB : à l'inverse des deux premiers pays, l'effort supplémentaire serait faible, voir nul en 2012 et plus conséquent en 2013. Les nouveaux plans d'économie en France s'élèveraient à 18 milliards en 2013. Dans le même temps, l'Allemagne ne devrait adopter de nouvelles mesures d'économies qu'en 2013, à hauteur de 34,6 milliards d'euros

Ces différents plans de rigueur nationaux, pris isolément, auraient un impact non négligeable sur la croissance des pays étudiés. En 2012, I'Allemagne et la France échap-

7. «La multiplication de la rigueur », Revue de l'OFCE, n 119, octobre 2011. 
peraient à une croissance négative (respectivement $0,3 \%$ et $0,0 \%$ ). Mais une telle stratégie plongerait I'Italie et l'Espagne dans une nouvelle et forte récession, avec un recul de leur PIB au-delà de -3,0 \% (tableau 6). En 2013 en revanche, la France et l'Allemagne basculeraient à leur tour en récession : les quatre grands pays de la zone euro afficheraient des taux de croissance négatifs allant de $-0,2 \%$ pour la France et $-0,4 \%$ pour l'Allemagne à $-2,0 \%$ pour l'Espagne

Bien entendu, si l'ensemble des grands pays de la zone euro venait à adopter la même stratégie en même temps alors l'effort d'économie serait supérieur. Sur les deux prochaines années, il s'élèverait à près de 4 points de PIB en Italie et en Espagne : plus précisément, il serait de 58 milliards d'euros en Italie (3,6 points de PIB), et 45,1 Md€ en Espagne (4,2 points de PIB). Cet effort supplémentaire serait de plus de 2 points de PIB en France et en Allemagne, représentant respectivement 43,0 et 61,0 Md€. Au total sur ces 4 pays étudiés, l'effort cumulé d'économie représenterait plus de $200 \mathrm{Md} €$ au cours de la période 2012-2013.

Le choc sur l'activité de ces pays serait alors puissant : il provoquerait en 2012 une récession violente pour certains pays avec une baisse du PIB de près de 4,0 \% (-4,2 \% en Italie et $-3,7 \%$ en Espagne). En 2013, la chute d'activité serait comprise entre $-1,1 \%$ en France et $-3,4 \%$ en Espagne.

Tableau 6. Impact sur le PIB du respect des engagements de réduction des déficits en 2012 et 2013

En \%

\begin{tabular}{|cccccccccc}
\hline \multicolumn{1}{c}{ DEU } & \multicolumn{3}{c}{ FRA } & \multicolumn{3}{c}{ ITA } & \multicolumn{3}{c}{ ESP } \\
& 2012 & 2013 & 2012 & 2013 & 2012 & 2013 & 2012 & 2013
\end{tabular}

Prévisions OFCE

Taux de croissance du PIB

$\begin{array}{llllllll}0,3 & 0,8 & 0,2 & 0,7 & -1,7 & -0,9 & -1,1 & -0,6\end{array}$

Solde public (en points de PIB)

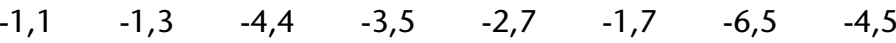

Effort supplémentaire à fournir si chaque pays respecte seul son engagement

$\begin{array}{lrrrrrrrr}\text { En milliards d'euros } & 5,2 & 34,6 & 0,0 & 18,0 & 32,0 & 13,0 & 22,5 & 10,8 \\ \text { En points de PIB } & 0,2 & 1,3 & 0,0 & 0,9 & 2,0 & 0,8 & 2,1 & 1,0 \\ \text { Taux de croissance du PIB } & 0,1 & -0,4 & 0,2 & -0,2 & -3,6 & -1,6 & -3,1 & -2,0 \\ \text { Solde public (en points de PIB) } & -1,0 & -0,5 & -4,5 & -3,0 & -1,6 & -0,1 & -5,3 & -3,0\end{array}$

Effort supplémentaire à fournir si les grands pays de la zone euro respectent tous leurs engagements

\begin{tabular}{lrrrrrrrr} 
En milliards d'euros & 15,7 & 45,3 & 8,0 & 28,0 & 38,5 & 19,5 & 26,8 & 18,3 \\
En points de PIB & 0,6 & 1,7 & 0,4 & 1,4 & 2,4 & 1,2 & 2,5 & 1,7 \\
Taux de croissance du PIB & $-0,7$ & $-1,2$ & $-0,7$ & $-1,1$ & $-4,2$ & $-2,5$ & $-3,7$ & $-3,4$ \\
Solde public (en points de PIB) & $-1,0$ & $-0,5$ & $-4,5$ & $-3,0$ & $-1,6$ & $-0,1$ & $-5,3$ & $-3,0$ \\
\hline
\end{tabular}

Sources : Prévisions et calculs OFCE.

La zone euro se retrouverait ainsi coincée dans cette logique de surenchère budgétaire et les agences de notation s'inquièteraient désormais du risque d'une récession profonde de la zone euro et de ses conséquences sur le système financier. La contagion s'accélèrerait et des grands pays de la zone euro seraient désormais dans la mire des marchés financiers et des spéculateurs. Les décisions du sommet européen du 
9 décembre 2011, visant à renforcer la discipline budgétaire, notamment par l'adoption de règles budgétaires contraignantes par les Etats de la zone euro, ne calmeraient pas les marchés financiers. Le renforcement seul de l'austérité budgétaire ne serait qu'un accélérateur de crise et ne ferait que renforcer le risque de disparition de la monnaie unique.

\section{Scénario 2 : un nouveau défaut dans la zone euro?}

Le second scénario à risque, qui n'est pas complètement indépendant du premier, est celui dans lequel un pays de la zone euro est confronté à un nouveau défaut sur sa dette publique, et dans le pire des cas à une sortie de la zone. Même dans un schéma de forte baisse du déficit public dans la zone euro, ce scénario ne peut pas écarté en raison des déséquilibres profonds qui persistent entre les pays membres en 2012 et 2013.

En effet, le rétablissement marqué des comptes publics de la zone euro d'ici à 2013 masque des situations extrêmement différenciées entre les pays membres, que ce soit en termes de finances publiques, de niveau de chômage ou des balances courantes (graphiques 4 et 5). Ces déséquilibres macroéconomiques ne seraient pas résorbés à I'horizon 2013 et pourraient conduire à des nouveaux épisodes de tensions, pouvant déboucher sur une nouvelle crise dans la zone euro.

Face à l'ampleur des déséquilibres, les pays qui présentent des comptes publics en bonne santé et des excédents commerciaux pourraient exiger des pays les plus déficitaires (budgétairement et commercialement) plus de rigueur. À défaut de transfert des pays excédentaires vers les pays déficitaires (notamment par la relance de la demande intérieure des pays présentant de forts excédents commerciaux), cette politique pourrait creuser la tombe des pays déjà en récession (Grèce, Portugal, Espagne et Italie), entraînant une nouvelle vague de chômage dans des pays déjà enlisés dans une crise sociale de grande ampleur. Les primes de risque sur les dettes souveraines pourraient repartir à la hausse et provoquer un nouveau cycle de crise de défiance au sein de la zone euro... Pour maintenir un haut niveau de solvabilité, les banques européennes, qui détiennent une masse importante de dettes souveraines des États de la zone euro, n'auront d'autre choix que de se délester des actifs publics dégradés pour éviter une baisse de leur ratio prudentiel.

Cette mécanique pro-cyclique pousserait les taux des pays en difficulté à la hausse renforçant le processus de défiance des investisseurs. Les banques qui encaissent des pertes face à la baisse de valeur des actifs dégradés et qui peuvent difficilement se recapitaliser sur les marchés seraient conduites à réduire la taille de leurs bilans en restreignant massivement le crédit bancaire, ce qui déboucherait sur un crédit crunch. Les effets négatifs de ce scénario seraient amplifiés si cette situation entraînait un nouveau défaut d'un pays de la zone euro ravivant les craintes fondées d'un risque systémique; Et dans le pire des cas, la sortie volontaire ou non d'un pays de la zone euro plongerait le système financier dans l'incertitude la plus totale qui déboucherait probablement sur une explosion de la zone euro et une crise monétaire mondiale. 
Qui sème la restriction récolte la récession : Perspectives économiques 2012-2013

Graphique 4. Solde public et balance courante pour les pays de la zone euro en 2011, 2012 et 2013

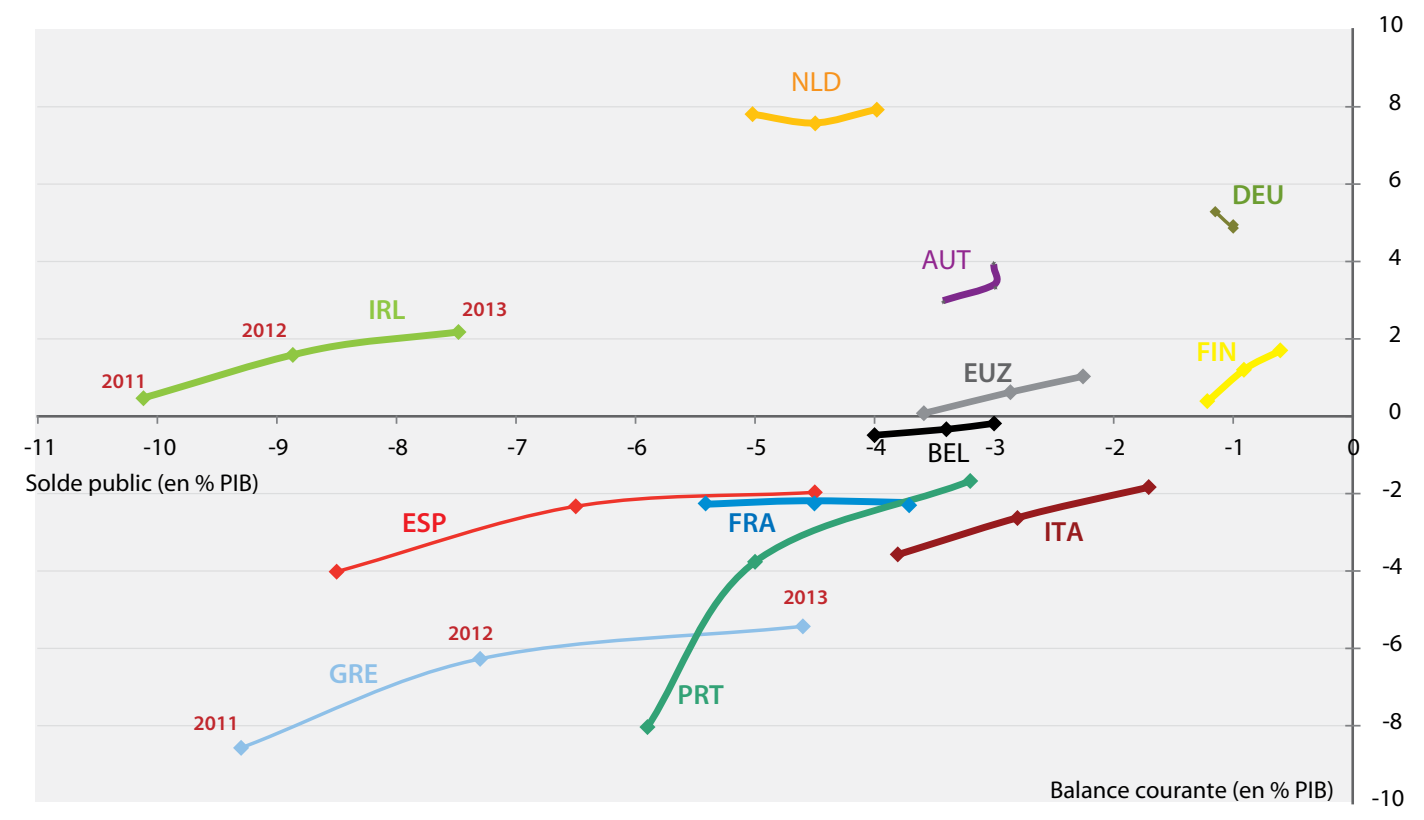

Sources : Prévisions OFCE, OCDE.

Graphique 5. Solde public et taux de chômage pour les pays de la zone euro en 2011, 2012 et 2013

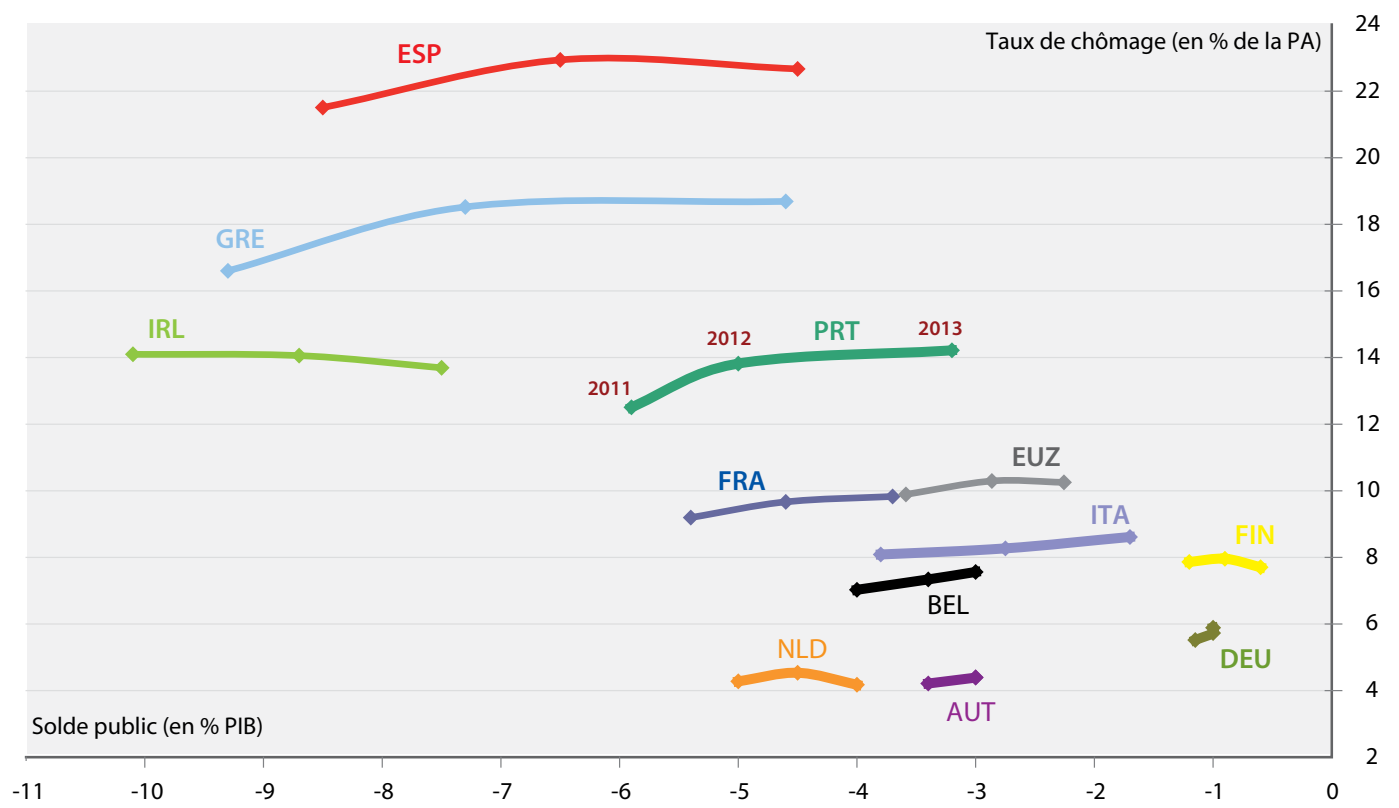

Sources : Prévisions OFCE, OCDE. 


\section{Scénario 3 : retour de balancier vers les pays anglo-saxons}

Si les scénarii 1 et 2 sont écartés, il est probable que le risque se déporte de la zone euro, si les sacrifices consentis $s^{\prime} y$ avèrent payant, vers $d^{\prime}$ autres zones (États-Unis et Royaume-Uni notamment). En effet, les pays industrialisés n'ayant pas fourni les efforts nécessaires pour redresser de façon significative leurs comptes publics pourraient se trouver dans le collimateur des marchés. Si l'on observe les évolutions attendues à I'horizon 2013 des tax gap - c'es-à-dire l'écart entre le solde public effectif et celui stabilise la dette publique en points de PIB - et des balances courantes dans la zone euro, au Royaume-Uni et aux États-Unis, les écarts diminuent peu, voire s'accroissent dans certains cas (graphique 6). En 2013, la zone euro devrait afficher à la fois un excédent de sa balance courante et pour la première fois depuis 2007 un tax gap positif qui annoncerait le début d'une décrue de sa dette publique (en points de PIB). À l'inverse, les États-Unis se retrouveraient dans une situation extrêmement inconfortable, affichant à la fois un déficit très élevé de leur balance courante et un tax gap encore largement négatif (environ -4 points de PIB), ce qui signifierait une nouvelle forte augmentation de la dette publique américaine. Le Royaume-Uni se situerait dans une situation intermédiaire avec une balance courante équilibrée mais un tax gap encore très négatif (environ -4 points de PIB).

Au regard de ces indicateurs, l'hypothèse d'un déplacement de la crise de la zone euro vers les États-Unis et le Royaume-Uni semble plus que probable. Si la capacité de résistance des États-Unis aux attaques des marchés financiers est sans commune mesure avec celle de la zone euro, il n'en reste pas moins que l'on pourrait assister à un fort mouvement de réallocation de l'épargne au détriment des États-Unis et du Royaume-Uni au profit de la zone euro. Dans ce contexte, le taux d'intérêt réel négatif sur la dette publique aux États-Unis et au Royaume-Uni deviendrait vite de I'histoire ancienne. Avec une prime de risque augmentant rapidement sur les titres publics de ces deux pays, les gouvernements n'auraient d'autre choix que de durcir fortement leurs politiques d'austérité, ce qui risquerait de faire basculer ces deux économies vers la récession avec des répercussions économiques et financières planétaires.

Dans aucun des trois scénarios la crise n'est réglée, les répliques pouvant surgir n'importe où et n'importe quand. 
Qui sème la restriction récolte la récession : Perspectives économiques 2012-2013

Graphique 6. Tax gap et balance courante en 2011, 2012 et 2013

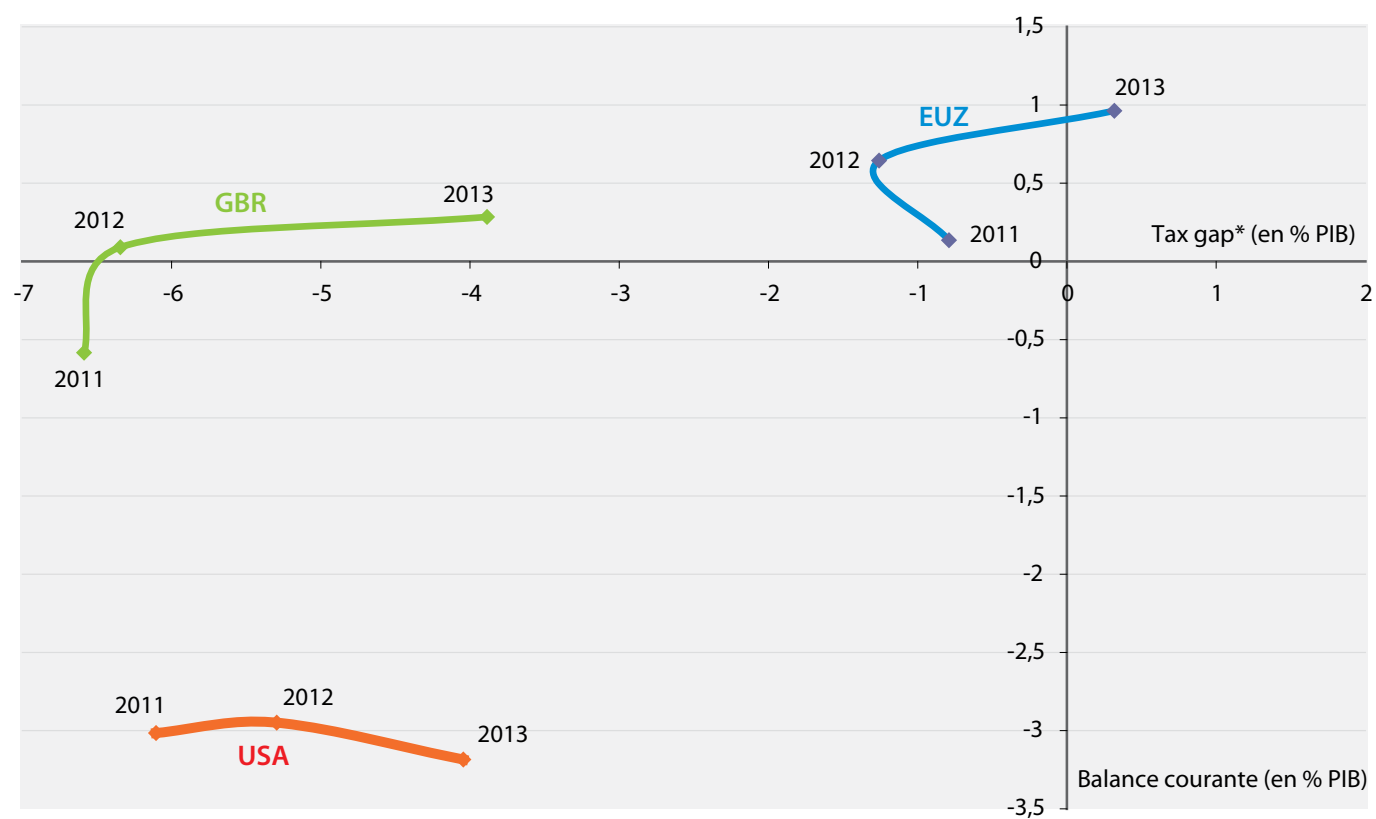

* Le tax gap est calculé comme l'écart entre le solde public effectif et celui qui stabilise la dette publique (en pts de PIB) à sa dernière valeur.

Sources : prévisions OFCE, OCDE 
Principales hypothèses de taux de change, taux d'intérêt et prix des matières premières

\begin{tabular}{|c|c|c|c|c|c|c|c|c|c|c|c|c|c|c|c|}
\hline & \multicolumn{4}{|c|}{2011} & \multicolumn{4}{|c|}{2012} & \multicolumn{4}{|c|}{2013} & \multirow{2}{*}{2011} & \multirow{2}{*}{2012} & \multirow{2}{*}{2013} \\
\hline & $\mathrm{T} 1$ & T2 & T3 & T4 & T1 & T2 & T3 & T4 & $\mathrm{T1}$ & T2 & T3 & T4 & & & \\
\hline \multicolumn{16}{|l|}{ Taux de change } \\
\hline $1 \$=\ldots$ Yen & 82,3 & 81,7 & 77,7 & 77,3 & 79,3 & 80,0 & 80,0 & 80,0 & 80,0 & 80,0 & 80,0 & 80,0 & 79,8 & 79,8 & 80,0 \\
\hline $1 £=\ldots$ euros & 1,17 & 1,13 & 1,14 & 1,17 & 1,20 & 1,20 & 1,18 & 1,18 & 1,16 & 1,15 & 1,15 & 1,15 & 1,15 & 1,19 & 1,15 \\
\hline Japon & 0,10 & 0,10 & 0,10 & 0,10 & 0,10 & 0,10 & 0,10 & 0,10 & 0,10 & 0,10 & 0,10 & 0,10 & 0,10 & 0,10 & 0,10 \\
\hline Zone euro & 1,00 & 1,22 & 1,47 & 1,31 & 1,00 & 1,00 & 1,00 & 1,00 & 1,00 & 1,00 & 1,00 & 1,00 & 1,25 & 1,00 & 1,00 \\
\hline Royaume-Uni & 0,50 & 0,50 & 0,50 & 0,50 & 0,50 & 0,50 & 0,50 & 0,50 & 0,50 & 0,50 & 0,50 & 0,50 & 0,50 & 0,50 & 0,50 \\
\hline \multicolumn{16}{|c|}{ Taux d'intérêt à 3 mois } \\
\hline Royaume-Uni & 0,81 & 0,85 & 0,88 & 1,00 & 1,04 & 1,05 & 1,05 & 1,05 & 1,05 & 1,05 & 1,05 & 1,05 & 0,88 & 1,05 & 1,05 \\
\hline \multicolumn{16}{|c|}{ Taux d'intérêt à 10 ans } \\
\hline États-Unis & 3,46 & 3,16 & 2,38 & 2,02 & 1,94 & 2,00 & 2,00 & 2,00 & 2,10 & 2,10 & 2,20 & 2,20 & 2,76 & 1,98 & 2,15 \\
\hline Japon & 1,25 & 1,18 & 1,05 & 1,01 & 0,98 & 1,00 & 1,00 & 1,00 & 1,10 & 1,10 & 1,10 & 1,10 & 1,13 & 0,99 & 1,10 \\
\hline Zone euro & 4,30 & 4,47 & 4,28 & 4,20 & 3,83 & 3,70 & 3,60 & 3,50 & 3,50 & 3,50 & 3,50 & 3,50 & 4,31 & 3,66 & 3,50 \\
\hline Royaume-Uni & 3,68 & 3,42 & 2,69 & 2,29 & 2,09 & 2,10 & 2,10 & 2,10 & 2,20 & 2,20 & 2,30 & 2,30 & 3,02 & 2,10 & 2,25 \\
\hline $\begin{array}{l}\text { Prix de pétrole, } \\
\text { Brent, en } \$\end{array}$ & 104,9 & 117,4 & 113,4 & 109,4 & 118,8 & 125,0 & 125,0 & 122,0 & 115,0 & 115,0 & 120,0 & 120,0 & 111,3 & 122,7 & 117,5 \\
\hline $\begin{array}{l}\text { Prix de pétrole, } \\
\text { Brent, en } €\end{array}$ & 76,8 & 81,6 & 80,3 & 81,2 & 90,7 & 97,7 & 100,0 & 97,6 & 89,8 & 88,5 & 92,3 & 92,3 & 80,0 & 96,5 & 90,7 \\
\hline
\end{tabular}

1. Moyenne sur la période.

2. Variation par rapport à la période précédente, en \%.

Sources : Taux de change et pétrole : relevé des cotations quotidiennes. Taux longs : T-Bond à 10 ans aux États-Unis, Benchmark à 10 ans au Japon, cours moyen des obligations d'État à 10 ans pour la zone euro, obligations d'État à 10 ans au Royaume-Uni. Matières premières industrielles : indice HWWA (Hambourg). Prévision OFCE mars 2012. 